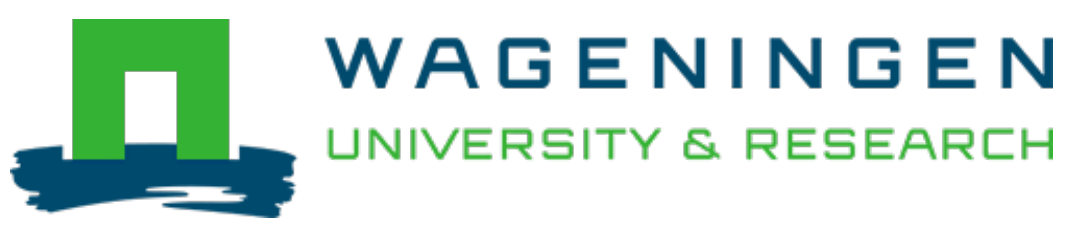

\author{
Evaluation of PBN spin-trapped radicals as early markers of lipid oxidation in \\ mayonnaise \\ Food Chemistry \\ Merkx, Donny W.H.; Plankensteiner, Lorenz; Yu, Yafei; Wierenga, Peter A.; Hennebelle, Marie et al \\ https://doi.org/10.1016/j.foodchem.2020.127578
}

This article is made publicly available in the institutional repository of Wageningen University and Research, under the terms of article $25 \mathrm{fa}$ of the Dutch Copyright Act, also known as the Amendment Taverne. This has been done with explicit consent by the author.

Article 25 fa states that the author of a short scientific work funded either wholly or partially by Dutch public funds is entitled to make that work publicly available for no consideration following a reasonable period of time after the work was first published, provided that clear reference is made to the source of the first publication of the work.

This publication is distributed under The Association of Universities in the Netherlands (VSNU) 'Article $25 \mathrm{fa}$ implementation' project. In this project research outputs of researchers employed by Dutch Universities that comply with the legal requirements of Article $25 \mathrm{fa}$ of the Dutch Copyright Act are distributed online and free of cost or other barriers in institutional repositories. Research outputs are distributed six months after their first online publication in the original published version and with proper attribution to the source of the original publication.

You are permitted to download and use the publication for personal purposes. All rights remain with the author(s) and / or copyright owner(s) of this work. Any use of the publication or parts of it other than authorised under article $25 \mathrm{fa}$ of the Dutch Copyright act is prohibited. Wageningen University \& Research and the author(s) of this publication shall not be held responsible or liable for any damages resulting from your (re)use of this publication.

For questions regarding the public availability of this article please contact openscience.library@wur.nl 


\title{
Evaluation of PBN spin-trapped radicals as early markers of lipid oxidation in mayonnaise
}

\author{
Donny W.H. Merkx ${ }^{\mathrm{a}, \mathrm{b}, \mathrm{c}}$, Lorenz Plankensteiner ${ }^{\mathrm{b}}$, Yafei Yu ${ }^{\mathrm{b}}$, Peter A. Wierenga ${ }^{\mathrm{b}}$, \\ Marie Hennebelle, ${ }^{\mathrm{b}, *}$, John P.M. Van Duynhoven ${ }^{\mathrm{a}, \mathrm{c}}$ \\ ${ }^{a}$ Unilever Food Innovation Centre, Bronland 14, 6708 WH Wageningen, The Netherlands \\ ${ }^{\mathrm{b}}$ Wageningen University \& Research, Laboratory of Food Chemistry, Bornse Weilanden 9, 6708 WG Wageningen, The Netherlands \\ ${ }^{\mathrm{c}}$ Wageningen University \& Research, Laboratory of Biophysics, Stippeneng 4, 6708 WE Wageningen, The Netherlands
}

\section{A R T I C L E I N F O}

\section{Keywords:}

ESR

PBN

Lipid oxidation

Bulk oil

Mayonnaise

MNP-L

${ }^{1} \mathrm{H}$ NMR

\begin{abstract}
A B S T R A C T
Quality deterioration of mayonnaise is caused by lipid oxidation, mediated by radical reactions. Assessment of radicals would enable early lipid oxidation assessment and generate mechanistic insights. To monitor short-lived lipid-radicals, $N$-tert-butyl- $\alpha$-phenylnitrone (PBN), a spin-trap, is commonly used. In this study, the fate of PBNadducts and their impact on lipid oxidation mechanisms in mayonnaise were investigated. The main signals detected by Electron Spin Resonance (ESR) were attributed to L--radicals attached to 2-methyl-2-nitrosopropane (MNP), one of three degradation products of the PBN-peroxy-adduct. The second degradation product, benzaldehyde, was detected with Nuclear Magnetic Resonance $\left({ }^{1} \mathrm{H}\right.$ NMR), in line with MNP-L adduct generation. For the third class of degradation products, LO--radicals, their scission products were detected with ${ }^{1} \mathrm{H}$ NMR and indicated that LO-radicals have a major impact on downstream oxidation pathways. This precludes mechanistical studies in presence of PBN. Degradation products of PBN-adducts can, however, be used for early assessment of antioxidants efficacy in oil-in-water emulsions.
\end{abstract}

\section{Introduction}

Lipid oxidation is one of the most detrimental reactions that can affect the quality of oil and oil-based emulsions. Lipid oxidation is a free radical chain reaction that is commonly divided into primary and secondary oxidation (Frankel, 2005). During primary oxidation, lipid-radicals $(\mathrm{L} \cdot)$ react with oxygen $\left(\mathrm{O}_{2}\right)$ to form peroxy-radicals $(\mathrm{LOO} \cdot)$ that can abstract a hydrogen from other lipids (LH), generating hydroperoxides ( $\mathrm{LOOH})$. Secondary oxidation is commonly described as the degradation of these hydroperoxides into aldehydes, epoxides and other secondary oxidation products. These mechanisms are well described in bulk oil systems (Schaich, 2012; Schaich, Xie, \& Bogusz, 2017). In oilin-water emulsions, the interfacial layer and transport of intermediates introduce an additional complexity to the lipid oxidation processes (Berton-Carabin, Ropers, \& Genot, 2014; Laguerre, Tenon, Bily, \& Birtić, 2020). Elucidation of these mechanisms ideally requires quantitative measures on both primary and secondary oxidation products. Currently, several methods can quantitatively monitor the generation of primary and secondary oxidation products, such as peroxide value, ThioBarbituric Acid Reactive Substances (TBARS), chromatographic methods (Barriuso, Astiasarán, \& Ansorena, 2013; Steenhorst-
Slikkerveer, Louter, Janssen, \& Bauer-Plank, 2000) and Nuclear Magnetic Resonance (NMR) (Merkx, Hong, Ermacora, \& van Duynhoven, 2018; Skiera et al., 2012). These approaches, however, focus on stable products and are not designed to capture the radical intermediates, which are critical components for mechanistical understanding.

A technique that is commonly used to detect and monitor radicals is Electron Spin Resonance (ESR). The radicals that are formed during lipid oxidation, however, are highly reactive with very low steady state concentrations, which prevent direct detection by ESR. For such analyses, spin-traps can be used, which are molecules that react with free radicals, effectively stabilizing the radicals for a sufficiently long time (Andersen \& Skibsted, 2006). The sensitivity of ESR is $\sim 1000$ times higher than NMR, potentially allowing oxidative assessment at even earlier time points. To investigate radicals in oil-in-water emulsions, the spin-trap should be stable at mildly accelerated shelf-life conditions, soluble in oil and should not be too expensive to be used in shelf-life studies. A spin-trap that meets these requirements is $N$-tert-butyl- $\alpha$ phenylnitrone (PBN). PBN is widely used for lipid oxidation studies (Boerekamp, Andersen, Jacobsen, Chronakis, \& García-Moreno, 2019; Cui, Lahti, \& Decker, 2016; Thomsen, Jacobsen, \& Skibsted, 2000; Thomsen, Kristensen, \& Skibsted, 2000; Thomsen, Vedstesen, \&

\footnotetext{
* Corresponding author.

E-mail address: Marie.Hennebelle@wur.nl (M. Hennebelle).
} 
Skibsted, 1999; Velasco, Andersen, \& Skibsted, 2004). Despite its wide use, its fate and potential impact on lipid oxidation mechanisms have not been investigated in detail. It has been reported that PBN rapidly degrades both in model systems at mild conditions (Janzen, Krygsman, Lindsay, \& Haire, 1990; Vicente et al., 1998), as well as in oil under extreme thermal stress $\left(180{ }^{\circ} \mathrm{C}\right)$ (Silvagni, Franco, Bagno, \& Rastrelli, 2010). Under these circumstances, PBN is reported to react with the $\mathrm{LOO} \cdot$ radicals, forming LOO-PBN, which rapidly decomposes to alkoxyradicals (LO·), benzaldehyde and 2-methyl-2-nitrosopropane (MNP) (Janzen et al., 1990; Silvagni et al., 2010). Here, MNP acts as a secondary spin-trap that reacts with $\mathrm{L} \cdot$ radicals to form the MNP-L adducts that are actually measured with ESR. At this point, whether this PBN degradation mechanism is the same in food emulsions in milder storage conditions has not been investigated. It would limit the applicability of spin traps for early diagnostics of lipid oxidation (J. Velasco, Andersen, \& Skibsted, 2005) and unravelling of lipid oxidation mechanisms.

In this work, we will first establish the PBN reaction pathways in rapeseed oil under modest shelf-life conditions. Next, these pathways will be verified in mayonnaise where the modulation of lipid oxidation mechanism by PBN will be studied. This will also involve the interaction of PBN reaction products with the antioxidant mechanisms of the metal chelator EDTA. Finally, we will assess the applicability and limits of using ESR for the early assessment of antioxidant efficacy.

\section{Material and methods}

\subsection{Materials}

$N$-tert-butyl-a-phenylnitrone (PBN, 99.5\%), 2-methyl-2-nitrosopropane dimer (MNP dimer) and 16-doxyl-stearic acid (16-DSA), ethylenediaminetetraacetic acid calcium disodium salt (CaNa ${ }_{2} \mathrm{EDTA}$ ), benzaldehyde (99\%) were purchased from Sigma Aldrich (Zwijndrecht, the Netherlands). $\mathrm{CDCl}_{3}$, DMSO- $d_{6}$ and $4 \AA$ molecular sieves were purchased from Euriso-top (Saint-Aubin, France). To facilitate solubility, PBN was ground before use using a pestle and mortar. Rapeseed (RP) oil, Medium Chain Triglyceride (MCT) oil, egg yolk, sodium chloride, sugar and starch were purchased from local suppliers. Alumina powder (MP EcoChrome ${ }^{\mathrm{TM}}$ ALUMINA N, Activity: Super I, Biomedicals) was purchased from MP Biomedicals (Irvine, USA).

\subsection{Rapeseed oil experiments}

PBN was directly dissolved in the rapeseed oil in a glass container. To speed up the dissolution, the oil was heated for $30 \mathrm{~min}$ at $30^{\circ} \mathrm{C}$. Two experimental series were performed on rapeseed oil samples. For the oxygen-availability series, $50 \mathrm{~mL}$ solutions of $40 \mathrm{mM}$ of PBN in rapeseed oil were prepared. The oil was separated into two $22 \mathrm{~mL}$ aliquots in $50 \mathrm{~mL}$ Falcon tubes (polypropylene). For the oxygen-rich sample, the Falcon tube was filled with atmospheric air and closed with a lid. For the oxygen-poor sample, the Falcon tube was bubbled continuously with nitrogen (no lid). The tubes were stored 7 days in a $50{ }^{\circ} \mathrm{C}$ water bath to accelerate the oxidation of the rapeseed oil. From both tubes, in duplicate, $1 \mathrm{~mL}$ aliquots were taken every day and frozen at $-80{ }^{\circ} \mathrm{C}$ until further use.

For the PBN-concentration series, $50 \mathrm{~mL}$ solutions of 10, 40 and $60 \mathrm{mM}$ of PBN in rapeseed oil were prepared. From this, $1 \mathrm{~mL}$ was transferred to $1.5 \mathrm{~mL}$ Eppendorf tubes (polypropylene) and stored in an oven at $40{ }^{\circ} \mathrm{C}$ up to 12 days. Samples were taken in duplicates at each time point and directly frozen at $-80{ }^{\circ} \mathrm{C}$ until further use.

\subsection{Mayonnaise production and storages}

All mayonnaises were prepared by using a Silverson mixer (Chesham, United Kingdom) for emulsification in $400 \mathrm{~g}$ batches. Rapeseed oil was used as such or stripped with alumina powder (MP EcoChrome $^{\mathrm{TM}}$ ALUMINA N, Activity: Super I, Biomedicals) to remove impurities and tocopherols (Berton, Ropers, Viau, \& Genot, 2011), this to prevent endogenous anti-oxidants interfering with the oxidation process. A $6.7 \mathrm{mM}$ EDTA stock solution was prepared by dissolving $250 \mathrm{mg}$ calcium disodium EDTA in $100 \mathrm{~mL}$ demineralized water. Two sets of experiment were performed with two types of formulations as described below.

\subsubsection{Quantitative assessment of radical formation in a model mayonnaise}

The mayonnaise samples were prepared with 0 or $0.2 \mathrm{mM}$ EDTA (respectively E- and E +) and contained 78\% of stripped rapeseed (SRP) oil. The SRP oil was devoid of native antioxidants and thus more prone to oxidation. The water phase $(82.4 \mathrm{~g})$ was prepared first. For this, $20 \mathrm{~g}$ egg yolk, $4.4 \mathrm{~g}$ sodium chloride, 0 or $3 \mathrm{~g}$ EDTA-solution and 14.5 or $11.5 \mathrm{~g}$ demineralized water were mixed in a $500 \mathrm{~mL}$ plastic container. Next, $312 \mathrm{~g}$ SRP oil was slowly added to form the emulsion and finally $5.6 \mathrm{~g}$ spirit vinegar was added to achieve the desired $\mathrm{pH}$ (3.8). To $120 \mathrm{~g}$ of both formulations (E- and E +), $1600 \mu \mathrm{L} 1.5 \mathrm{M}$ PBN in DMSO (details in SI1) was post-added to prepare $20 \mathrm{mM}$ PBN formulations (E-P + and $\mathrm{E}+\mathrm{P}+$ ). Aliquots of $1 \mathrm{~g}$ were stored in $20 \mathrm{~mL}$ screwcap vials in the dark at $40{ }^{\circ} \mathrm{C}$. Aliquots were removed from storage in duplicate for a total of 57 days with a high sampling density during the first ten days, since we expected to see most PBN-activity during the early oxidation phase. The aliquots were then stored at $-20{ }^{\circ} \mathrm{C}$ until further analysis. For all samples, ${ }^{1} \mathrm{H}$ NMR was used to monitor the generation of LOOH and $n$ alkanals, as representatives for respectively primary and secondary lipid oxidation. MNP-L spin-adduct and benzaldehyde generation were respectively measured by ESR and ${ }^{1} \mathrm{H}$ NMR for the samples with PBN.

\subsubsection{Early assessment of antioxidant protection against primary oxidation} in a full mayonnaise.

For the assessment on the oxidative stability in mayonnaises, mayonnaise samples were prepared with four levels of EDTA $(0,0.03$, 0.06 and $0.20 \mathrm{mM})$ and a $65 \%$ oil-content. The water phase $(113.6 \mathrm{~g})$ was prepared first. For this, $16.8 \mathrm{~g}$ egg yolk, $4.4 \mathrm{~g}$ sodium chloride, $4 \mathrm{~g}$ starch, $5.2 \mathrm{~g}$ sugar, 0 to $3 \mathrm{~g}$ EDTA-solution and 24.8 to $21.8 \mathrm{~g}$ demineralized water were mixed in a $500 \mathrm{~mL}$ plastic container. Next, $260 \mathrm{~g}$ rapeseed oil was slowly added to form the emulsion and finally $10.4 \mathrm{~g}$ spirit vinegar was added to achieve the desired $\mathrm{pH}$ (3.8). For the NMRassessment, aliquots of $1 \mathrm{~g}$ were stored in $20 \mathrm{~mL}$ screwcap vials at $50{ }^{\circ} \mathrm{C}$. Aliquots were removed from storage in duplicate at set intervals $(0,1,4$ and 6 days) and stored at $-20{ }^{\circ} \mathrm{C}$ until further analysis. For the ESRassessment, $50 \mu \mathrm{L}$ of $700 \mathrm{mM}$ PBN solution (PBN in MCT oil, details in SI1) was added to $5 \mathrm{~g}$ aliquots, in triplicate for each formulation, in $15 \mathrm{~mL}$ Falcon tubes (polypropylene) and gently stirred. The mayonnaises were incubated at $50{ }^{\circ} \mathrm{C}$ for $5 \mathrm{~h}$. During incubation, aliquots of mixtures were collected in triplicate at set intervals $(0,1.5,3$ and $5 \mathrm{~h})$ and stored at $-80{ }^{\circ} \mathrm{C}$ until further analysis.

\subsection{Electron spin Resonance (ESR)}

Frozen mayonnaise samples were thawed at room temperature, resulting in separation between the oil and the water phase. The sample (oil or oil-layer of the mayonnaise) was transferred to a $200 \mu \mathrm{L} \mathrm{mi-}$ cropipette capillary to a minimal filling height of $4 \mathrm{~cm}$. The capillary was placed in a quartz ESR tube of $5 \mathrm{~mm}$ diameter and subsequently moved into the ESR cavity. The ESR spectra were recorded with an Xband ESR spectrometer (Elexsys E 500, Bruker, Rheinstetten, Germany) running at $9.32 \mathrm{GHz}$. For the acquisition, the microwave power was set at $13.72 \mathrm{~mW}$, the modulation amplitude at $3.0 \mathrm{G}$, with the center field at $3328 \mathrm{G}$ and a sweep width of $100 \mathrm{G}$. 1024 number of points were used with both a conversion time and time constant of 164 . The acquisition settings were kept constant for all quantification measurements, only the receiver gain was adjusted $(20-60 \mathrm{~dB})$ and the outcome of the measurement was accordingly corrected. Generally, spectra were recorded at room temperature. For the identification of the spin adducts, the spectra were recorded at $70{ }^{\circ} \mathrm{C}$ in order to increase the 
molecular mobility (Thomsen et al., 1999) and the modulation amplitude was reduced up to 0.04 to prevent spectral line broadening. The trapped radicals were quantified with a calibration curve $(0-5 \mathrm{mM})$ of the stable radical 16-DSA. The analysis of the ESR spectra was carried out with the software package Easyspin (Stoll \& Schweiger, 2006), running in a MATLAB (version R2017b, MathWorks, USA) environment. The spectra were imported and after applying a smoothing function, the double integration was performed. Detected spin adducts were only quantified if the signal to noise ratio was higher than 10 .

\subsection{Nuclear Magnetic Resonance (NMR)}

For the quantification of oxidation products, the (separated) oil was dissolved in a $5: 1 \mathrm{CDCl}_{3}:$ DMSO- $d_{6}$ solvent. For the benzaldehyde assignment, $25 \mu \mathrm{L}$ pure benzaldehyde was added to $600 \mu \mathrm{L}$ of the mixture. Single pulse and band selective ${ }^{1} \mathrm{H}$ NMR spectra were recorded on a $600 \mathrm{MHz}$ (14.1 T) Bruker Avance III NMR spectrometer (Bruker BioSpin, Switzerland) according to the protocol described in earlier work (Merkx et al., 2018). The spectrum obtained by single pulse excitation was used for the quantification of PBN $(\delta 8.3 \mathrm{ppm})$, triglyceride $(\delta 4.4 \mathrm{ppm})$ and optionally benzaldehyde $(\delta 7.5 \mathrm{ppm})$. The triglyceride (TG) peak area was used for the internal calibration for the quantification of the hydroperoxides, aldehydes and benzaldehyde. For the band selective experiment, the region between $\delta 13.0$ and $8.0 \mathrm{ppm}$ was selectively excited. Hydroperoxides, aldehydes and benzaldehyde were quantified via integration of the signal regions between $\delta$ $11.2-10.7 \mathrm{ppm}, \delta 9.8-9.2 \mathrm{ppm}$ and $\delta 10.1-9.9 \mathrm{ppm}$, respectively. Details on calculations of concentrations from the integral regions in the single pulse and selective excitation experiment are described in previous work (Merkx et al., 2018).

\subsection{Statistical analysis}

ANOVA tests were performed, followed by Bonferroni corrected two-tailed T-tests to assess the significance of the differences between samples and differences over time. All tests were performed using Microsoft Excel. Statistical significance was set at $\mathrm{p}<0.05$.

\section{Results and discussion}

\subsection{Identification and quantification of spin-trapped radicals in rapeseed oil}

The mechanism of PBN spin trapping was first investigated in pure rapeseed oil. Rapeseed oil was the main ingredient of mayonnaise and its use simplified the sample handling and reduced the mechanistic complexity. This trial was done using two oxygen conditions (i.e. oxygen-rich and oxygen-depleted) to explore the PBN spin trapping mechanisms in the most opposing scenarios (Silvagni et al., 2010) in mild storage conditions. Under oxygen rich conditions, an ESR signal was observed after $24 \mathrm{~h}$ at $50{ }^{\circ} \mathrm{C}$. Three hyperfine lines $\left(\mathrm{I}_{\mathrm{N}}=1\right)$ with a hyperfine coupling constant $\left(\mathrm{a}_{\mathrm{N}}\right)$ of $14.8 \mathrm{G}$ were clearly distinguished (Fig. 1A). This coupling constant $(14.8 \mathrm{G})$ suggests the presence of a nitroxyl group. After increasing the temperature in the ESR tube to $70{ }^{\circ} \mathrm{C}$ to increase the molecular mobility, the three hyperfine lines were resolved into six hyperfine lines. A second hyperfine interaction was visible with a hyperfine coupling constant $\left(\mathrm{a}_{\mathrm{H}}\right)$ of $2.1 \mathrm{G}$ (Fig. 1B). This combination of hyperfine coupling constants corresponds to MNP-L adducts (Vicente et al., 1998). Importantly, no superposition of hyperfine splittings that could indicate the presence of other spin adducts (e.g. MNP-OOL or PBN-L) were observed in the ESR-spectra. The sample was cooled down and measured again to verify that short heating to $70{ }^{\circ} \mathrm{C}$ did not irreversibly generate a new spin adduct. Here, the same spectrum as before the heating was observed (data not shown), indicating that the same molecule was measured at room temperature and at $70{ }^{\circ} \mathrm{C}$. To confirm the identification of MNP-L
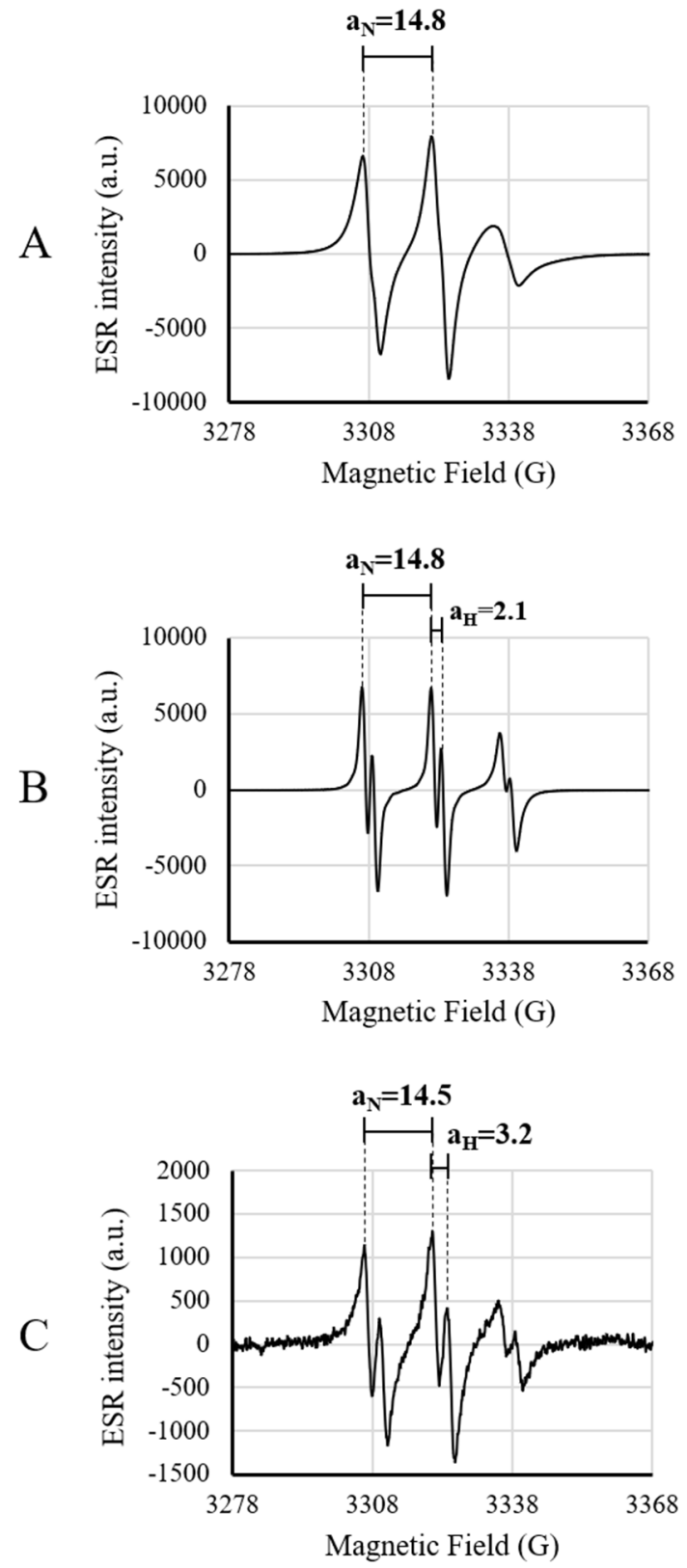

Fig. 1. ESR spectra of spin adducts in rapeseed oil after PBN addition ( $40 \mathrm{mM})$ and heated storage $\left(50{ }^{\circ} \mathrm{C}\right.$ for 7 days). A: ESR spectrum of rapeseed oil stored at $50{ }^{\circ} \mathrm{C}$ under aerobic conditions and measured at room temperature; B: ESR spectrum of rapeseed oil stored at $50{ }^{\circ} \mathrm{C}$ under aerobic conditions and measured at $70{ }^{\circ} \mathrm{C}$; C: ESR spectrum of nitrogen bubbled rapeseed oil stored at $50{ }^{\circ} \mathrm{C}$ and measured at room temperature.

adducts, MNP-dimer was added to rapeseed oil and stored at $40{ }^{\circ} \mathrm{C}$. As expected, the MNP-adducts that were observed with ESR showed identical hyperfine splittings to those described above (Figure SI2).

Under oxygen depleted conditions, the ESR spectra (Fig. 1C) clearly showed a different hyperfine splitting than the one observed under oxygen rich condition (Fig. 1A/B). The coupling constants were $14.5 \mathrm{G}$ and $3.2 \mathrm{G}$ for respectively $\mathrm{a}_{\mathrm{N}}$ and $\mathrm{a}_{\mathrm{H}}$. These coupling constants were assigned to the spin adducts between PBN and alkyl radicals (PBN-L) (Vicente et al., 1998). This observation matched with our hypothesis that under oxygen poor conditions, no PBN-OOL spin adducts were generated that could decompose to form MNP and subsequently MNP-L adducts, as observed under oxygen rich condition.

The previously reported degradation mechanisms of PBN-OOL to MNP implied the simultaneous formation of benzaldehyde (Janzen 
et al., 1990). Hence, we verified the appearance of benzaldehyde by ${ }^{1} \mathrm{H}$ NMR. In the ${ }^{1} \mathrm{H}$ NMR spectrum of rapeseed oil with $40 \mathrm{mM}$ PBN, a carbonyl proton signal was detected at $\delta 10.15 \mathrm{ppm}$. To verify the assignment of the carbonyl peak to benzaldehyde, we performed a spiking experiment with pure benzaldehyde and a 2D TOCSY experiment (Assignment in SI3). The signal of the carbonyl proton of benzaldehyde ( $\delta$ $10.15 \mathrm{ppm}$ ) resonated between the hydroperoxide ( $\mathrm{LOOH})$ region and aldehyde regions. This allowed for benzaldehyde quantification ( $\mathrm{LoQ}=0.05 \mathrm{mM}$ ) using the same band-selective pulse used for exciting the $\mathrm{LOOH}$ and aldehyde regions, as described in our previous work (Merkx et al., 2018).

Combining assessments of MNP-L or PBN-L by ESR and benzaldehyde by ${ }^{1} \mathrm{H}$ NMR allowed for a multifaceted approach to monitor the fate of PBN-OOL. After 7 days under oxygen-depleted conditions, around $2 \mu \mathrm{M}$ of PBN-L and no benzaldehyde were detected. In the absence of oxygen, little to no LOO - radicals were formed to consequently form benzaldehyde (via PBN-OOL). This enabled PBN to directly react with the L - to form PBN-L in low amounts. Under oxygen-rich conditions, concentrations of $360 \mu \mathrm{M}$ MNP-L and $800 \mu \mathrm{M}$ benzaldehyde was reached (Figure SI4). The benzaldehyde and MNP-L levels after $168 \mathrm{~h}$ differed by approximately a factor of two. This difference was attributed to higher stability of benzaldehyde than MNP-L. Benzaldehyde was not expected to degrade within these $168 \mathrm{~h}$, whereas MNP-L is a radical species that is prone to reduction and consequently a loss of ESR signal. Moreover, the MNP-L spin adducts showed a lag time of $24 \mathrm{~h}$ compared to benzaldehyde. Indeed, in order to form the MNP-L spin adducts, MNP needed to compete with $\mathrm{O}_{2}$ to react with $\mathrm{L} \cdot$.

This experiment showed that under mild storage conditions, MNP-L is indeed the spin-adduct that is detected with ESR when PBN was initially added (Fig. 2). The MNP-L adducts are a product of the free alkyl radicals $(\mathrm{L} \cdot)$ with MNP, which in turn is a decomposition product of the PBN-OOL adducts. Benzaldehyde, the by-product of PBN-OOL decomposition can be quantified by ${ }^{1} \mathrm{H}$ NMR and thereby can be used as a direct marker and proof of PBN-OOL decomposition and as an indirect marker of $\mathrm{LOO} \cdot$-generation.

Next, the effect of the PBN concentration on the formation of the MNP-L adducts and benzaldehyde was evaluated. As expected, in absence of PBN, no MNP-L and benzaldehyde were formed. In the first $200 \mathrm{~h}$, increasing the PBN concentration up to $60 \mathrm{mM}$ PBN, resulted in a proportional increase of MNP-L and benzaldehyde to respectively $0.16 \mathrm{mM}$ and $0.25 \mathrm{mM}$ (Fig. 3). Thus, the total amount of MNP-L and benzaldehyde depended on the concentration of added PBN. This dependence should be considered when interpreting MNP-L and benzaldehyde concentrations. Furthermore, it complicates the quantitative comparison between experimental series with different PBN concentrations. More importantly, the increase in the amount of MNP and benzaldehyde with increasing PBN concentration is likely accompanied by an increase in $\mathrm{LO}$ - concentration, which can contribute to the generation of more $\mathrm{L} \cdot$ and $\mathrm{LOO} \cdot$, and consequently of more PBN-OOL, MNP and benzaldehyde. This concentration-dependence clearly shows that PBN addition perturbs the oxidative reaction mechanism in rapeseed oil. This limits the applicability of spin-trapping as quantitative tool to link the measured spin adducts to other oxidation products, such as LOOHs and aldehydes for e.g. mass balancing purposes.

\subsection{Quantitative assessment of radical formation in mayonnaise}

Next, we verified that the radical trapping and adduct degradation mechanisms observed in oil also occurred in mayonnaises. We analysed benzaldehyde generation and PBN consumption in an accelerated storage trial of mayonnaise $\left(50{ }^{\circ} \mathrm{C}, 200 \mathrm{~h}\right)$ with ${ }^{1} \mathrm{H}$ NMR. In mayonnaise with $20 \mathrm{mM}$ PBN, the consumption of PBN was accompanied by generation of an equivalent amount of benzaldehyde (Figure SI5). Furthermore, MNP-L adducts were detected with ESR (data not shown). This confirmed that also in the more complex mayonnaise system, PBN decomposes to benzaldehyde and MNP via the PBN-OOL intermediate.

Since the general mechanism of the PBN spin-trapping has a major impact on the fate of radicals, we investigated this influence in mayonnaises prepared with and without EDTA. Without PBN, both the LOOH (Fig. 4A) and aldehyde generation (Fig. 4B) was higher in the absence of EDTA than in its presence. Different trends were observed when PBN was present in the mayonnaises. Firstly, PBN significantly $(\mathrm{p}<0.05)$ inhibited $\mathrm{LOOH}$ generation, both for $\mathrm{E}-(37 \mathrm{mM}(\mathrm{P}+)$ and $2 \mathrm{mM}(\mathrm{P}-)$ at $10 \mathrm{~d})$ and $\mathrm{E}+(12.5(\mathrm{P}+)$ and $8.3 \mathrm{mM}(\mathrm{P}-)$ at $21 \mathrm{~d})$. Moreover, in the E-P +, the initial LOOH concentrations significantly ( $p<0.05$ ) decreased instead of up in the first two days (from $1.7 \mathrm{mM}$ to $0.6 \mathrm{mM}$ in the first $48 \mathrm{~h}$, Figure SI6). Secondly, the generation of $n$ alkanals was significantly higher $(\mathrm{p}<0.05)$ in the presence of PBN, for both $\mathrm{E}-(3.0 \mathrm{mM}(\mathrm{P}+)$ and $0.5 \mathrm{mM}(\mathrm{P}-)$ at $21 \mathrm{~d})$ and for the $\mathrm{E}$ + samples $(2.1 \mathrm{mM}(\mathrm{P}+)$ and $0.1 \mathrm{mM}(\mathrm{P}-)$ at $21 \mathrm{~d})$. This observation was especially surprising on $\mathrm{E}+$ samples, which barely showed any $n$ alkanal generation in the absence of PBN (E $+\mathrm{P}-)$, but high concentrations in its presence $(\mathrm{E}+\mathrm{P}+)$. For both $\mathrm{E}+\mathrm{P}+$ and $\mathrm{E}-\mathrm{P}+$, the $\mathrm{PBN}$ was consumed $\left(<1 \mathrm{mM}\right.$ ) after 28 days (data not shown, measured with ${ }^{1} \mathrm{H}$

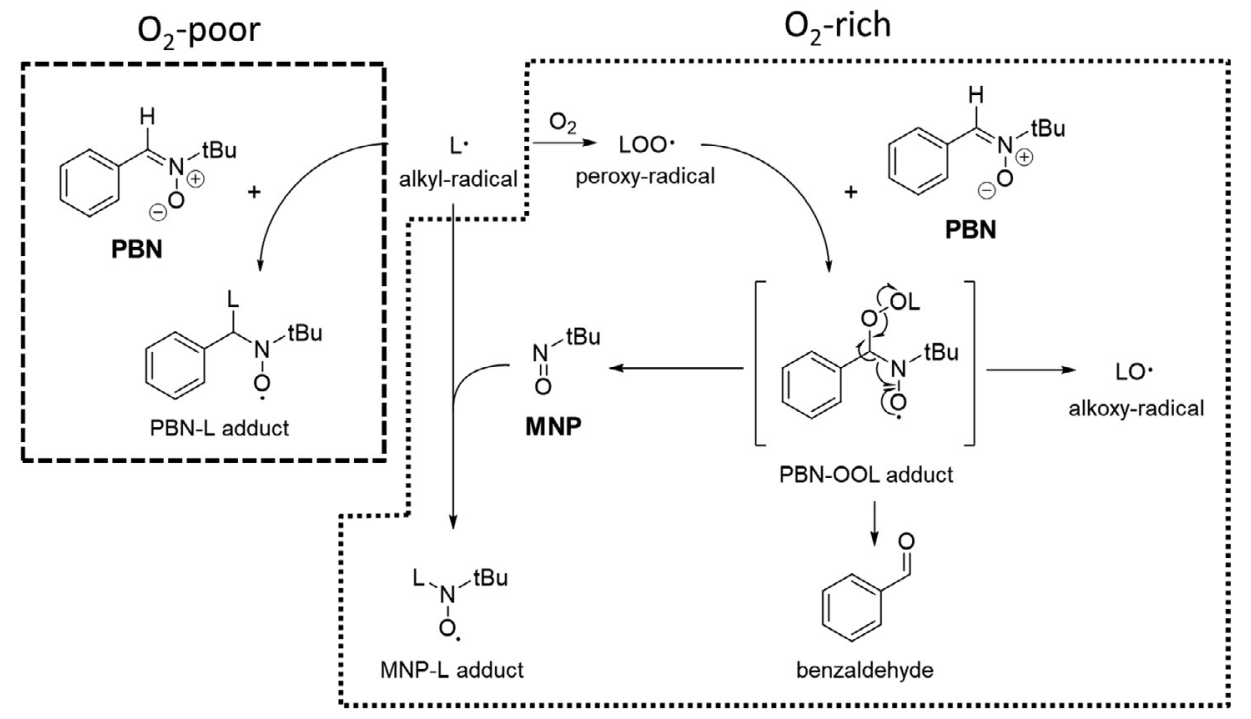

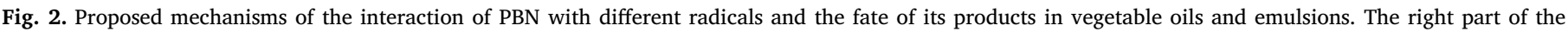
diagram is in oxygen rich conditions, the left part under oxygen depleted conditions. 
A

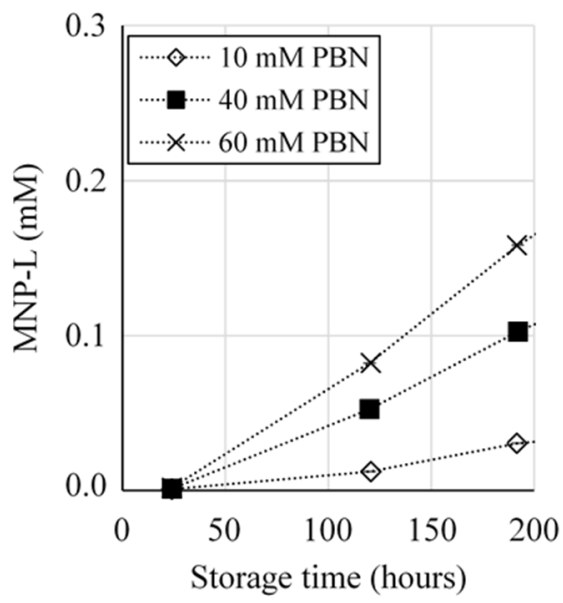

B

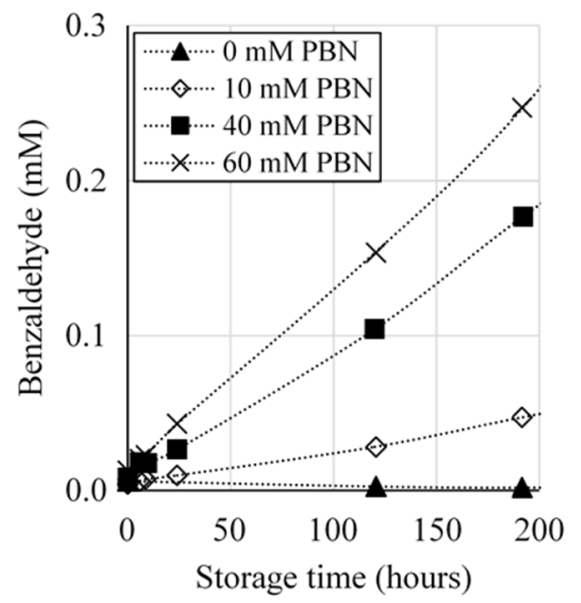

Fig. 3. Formation of MNP-L spin adducts (A) and benzaldehyde (B) during the storage of rapeseed oil at $40{ }^{\circ} \mathrm{C}$ : free radicals were trapped with different concentrations of PBN; … $0.0 \mathrm{mM}, \ldots . .10 \mathrm{mM}$, ……… $40 \mathrm{mM}, \ldots \times 60 \mathrm{mM}$; values are shown as mean $(\mathrm{n}=2)$. Lines are drawn with purpose of guiding the eye.
NMR).

The difference between the $\mathrm{LOOH}$ concentrations observed in mayonnaises with and without PBN was attributed to the reactivity of PBN with LOO-, effectively inhibiting the LOOH-propagation and instead generating the PBN-OOL intermediate. As discussed in section 3.1, the PBN-OOL intermediate is labile under present circumstances, and readily formed MNP, benzaldehyde and $\mathrm{LO}$ - radicals. Both the MNP-L and benzaldehyde concentrations (Fig. 4C/D) were measured with ESR and NMR and increased significantly ( $p<0.05)$, already in the first days/hours. The LO - radicals were not directly detected, but are known to undergo beta-scission, which is the main driver for the generation of aldehydes. This would explain that upon addition of PBN to mayonnaises, we observed an increase of $n$-alkanals, benzaldehyde and MNP-L adducts.

When EDTA was present and PBN absent (E+P-), both primary oxidation (LOOHs) and secondary oxidation (n-alkanals) products were lower than when both EDTA and PBN were absent (E-P-). Here, EDTA worked as expected as a secondary antioxidant through the chelation of iron to inhibit the catalysing effect of this transition metal. The redox $\mathrm{Fe}^{2+} / \mathrm{Fe}^{3+}$ cycling catalytic action is reported to go primarily via existing LOOHs (Laguerre et al., 2020; Merkx, Delić, Wierenga, Hennebelle, \& van Duynhoven, 2019; Mozuraityte, Rustad, \& Storro, 2008). These LOOHs could decompose to form LOO - and LO radicals, which will respectively fuel the propagation of $\mathrm{LOOH}$ generation and form aldehydes via beta-scission of $\mathrm{LO}$ - (Fig. 5). In the presence of PBN, the LOO - radicals can react with both PBN and LH to form respectively the LOO-PBN intermediates and LOOHs. In the presence of both PBN and EDTA $(\mathrm{E}+\mathrm{P}+)$, there is little to no free iron (or any other transition metal) in the mixture to catalyse the decomposition of $\mathrm{LOOH}$. Therefore, the accumulated LOOHs will not degrade, which results in a steady increase of LOOHs. However, in the absence of EDTA (E-P +), free transition metals will be present and catalyse the decomposition of LOOHs to LOO - and LO radicals. This means that for E-P +, contrary to $\mathrm{E}+\mathrm{P}+$, LOOHs will be degraded much more rapidly, which explains the initial decrease of $\mathrm{LOOH}$ in E-P + and why the LOOH levels are very low until the moment PBN was consumed ( $~ 28$ days). Furthermore, the first 28 days, the $n$-alkanal levels (a beta-scission product of LO·) are significantly $(\mathrm{p}<0.05)$ higher for the E-P + than the $\mathrm{E}+\mathrm{P}+$.

\subsection{Early assessment of antioxidant protection against primary oxidation in mayonnaise}

PBN spin trapping has a significant impact on lipid oxidation mechanisms, but whether PBN could be used as an early oxidative assessment tool was still undisclosed. In the experiments discussed in 3.2, the MNP-L concentration started to decrease after eight days and was no longer detectable by ESR after 50 days (Figure SI6). In the first five days, MNP-L concentrations were already more than ten times lower than the benzaldehyde concentrations (Fig. 4C/D), which hinted at a considerable MNP-L reduction in mayonnaises compared to oil (section 3.1). Nonetheless, MNP-L formation (Fig. 4D) was clearly delayed in the presence of EDTA $(\mathrm{E}+\mathrm{P}+)$ compared to the mayonnaise without EDTA $(\mathrm{E}-\mathrm{P}+)$. This suggested that MNP-L generation can be used as an early indicator for lipid oxidation in mayonnaise.

The applicability of PBN-spin trapping for early assessment of oxidative stability lipid oxidation in mayonnaises was verified in a trial with mayonnaises with four different levels of EDTA stored at $50{ }^{\circ} \mathrm{C}$ with a PBN concentration of $10 \mathrm{mM}$. Lowering the PBN concentration (i.e. from $10 \mathrm{mM}$ to $1 \mathrm{mM}$ ) might have a lower perturbing effect on the oxidative mechanism, with the drawback of less MNP-L generation in the process and was therefore not investigated at this point. Increasing EDTA concentrations were associated with a decrease in the formation of MNP-L adducts, as measured with ESR. Within five hours, the different formulations could be clearly significantly $(p<0.05)$ ranked based on their MNP-L levels (Fig. 6A). Such a distinction between the formulations was possible only after 4 days when considering the inhibition of LOOH formation measured by ${ }^{1} \mathrm{H}$ NMR. Moreover, at room temperature, MNP-L assessment by ESR allowed oxidative ranking after five days (Figure SI7, no ${ }^{1} \mathrm{H}$ NMR data). Thus, although PBN addition significantly altered the oxidative mechanism, it has the potential to be used as a tool to rank the oxidative stability of emulsions via the determination of the MNP-L adducts.

\section{Conclusions}

In mayonnaise, already at mild $\left(=<50{ }^{\circ} \mathrm{C}\right)$ shelf-life conditions, the PBN-OOL adducts rapidly degrades to MNP, benzaldehyde and alkoxy-radicals. The observed ESR-signal predominantly originates from the adducts of MNP and alkyl radicals. Benzaldehyde concentrations can be determined by ${ }^{1} \mathrm{H}$ NMR and used for estimating the cumulative amount of LOO - that has been trapped by PBN. Hence, both NMR and ESR can be used in combination with PBN to assess LOO formation in early (hours-days) stages of lipid oxidation. The trapping of LOO - radicals and subsequent generation of $\mathrm{LO}$ - radicals impacts downstream reaction pathways, which precludes mechanistic studies in the presence of PBN. PBN spin-trapping in combination with ESR and NMR detection does allow for assessment of antioxidant protection against primary oxidation at mild conditions. 

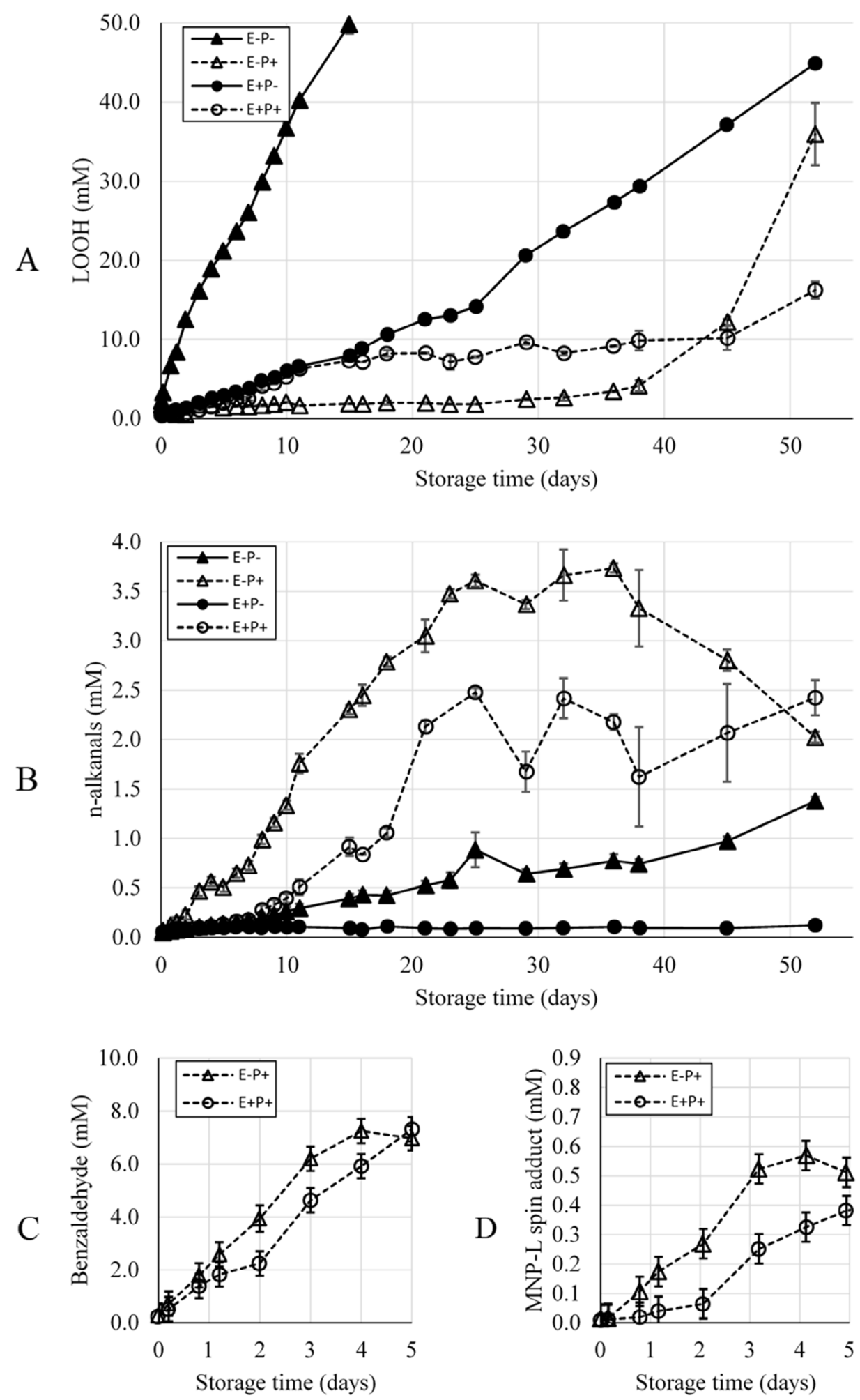

Fig. 4. Oxidation products, $\mathrm{PBN}$ and benzaldehyde concentrations as a function of storage time at $40{ }^{\circ} \mathrm{C}$. A: Sum of hydroperoxides by ${ }^{1} \mathrm{H}$ NMR, B: $n$-alkanals by ${ }^{1} \mathrm{H}$ NMR, C: benzaldehyde by ${ }^{1} \mathrm{H}$ NMR (first 5 storage days, only P+), D: MNP-L by ESR (first 5 storage days only P +). The investigated mayonnaise systems: with PBN ( + + open symbols), without PBN (P-, closed symbols), with EDTA (E + circles) and without EDTA (E-, triangles). Values are shown as mean ( $\mathrm{n}=2$ for ${ }^{1} \mathrm{H}$ NMR, $\mathrm{n}=1$ for ESR (standard error $0.05 \mathrm{mM}$ )), with the error bars displaying the standard deviation. Lines are drawn with purpose of guiding the eye.

\section{CRediT authorship contribution statement}

Donny W.H. Merkx: Investigation, Validation, Conceptualization, Writing - original draft. Lorenz Plankensteiner: Investigation, Methodology, Software. Yafei Yu: Methodology, Validation. Peter A.
Wierenga: Conceptualization, Software. Marie Hennebelle: Supervision, Writing - review \& editing. John P.M. Van Duynhoven: Conceptualization, Supervision, Writing - review \& editing. 


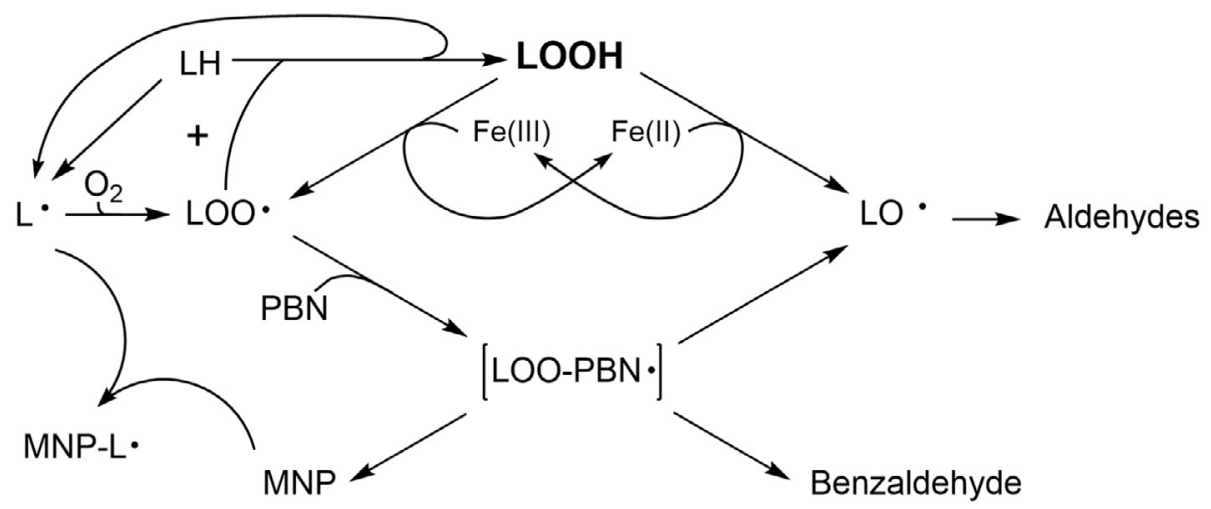

Fig. 5. Schematic overview of modulation of lipid oxidation pathways in mayonnaise by PBN spin-trapping.

A

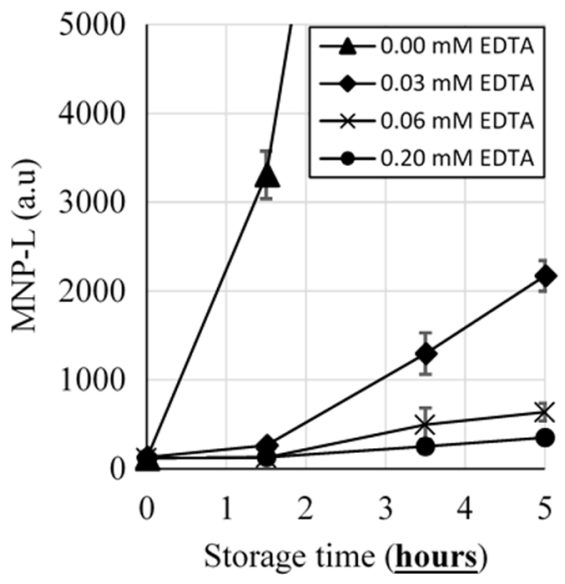

B

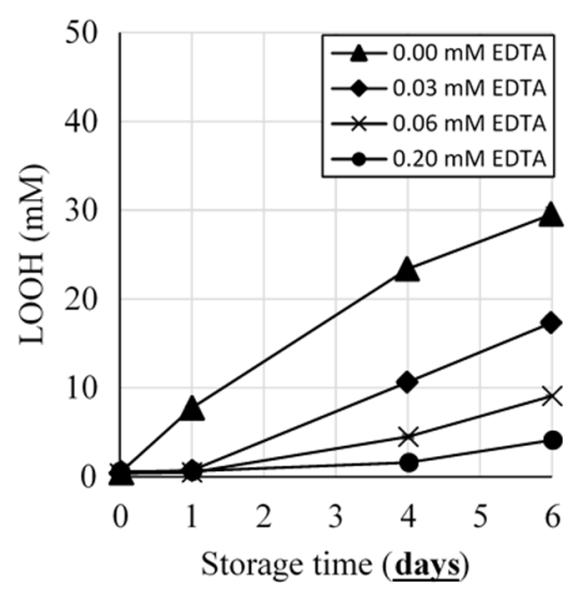

Fig. 6. MNP-L and hydroperoxides in mayonnaises as a function of different storage times at $50{ }^{\circ} \mathrm{C}$. A: relative MNP-L concentration in the presence of $10 \mathrm{mM}$ PBN, B: absolute LOOH concentrations in the absence of PBN. The four lines represent: $0 \mathrm{mM}$ EDTA (־), $0.03 \mathrm{mM}$ EDTA $(\multimap-), 0.06 \mathrm{mM}$ EDTA $(\multimap \leftarrow)$ and $0.2 \mathrm{mM}$ EDTA $(\multimap-)$.

\section{Declaration of Competing Interest}

The authors declare that they have no known competing financial interests or personal relationships that could have appeared to influence the work reported in this paper.

\section{Acknowledgements}

Elena Golovina and John Phillipi are gratefully acknowledged for their help in setting up the ESR method.

\section{Appendix A. Supplementary data}

Supplementary data to this article can be found online at https:// doi.org/10.1016/j.foodchem.2020.127578.

\section{References}

Andersen, M. L., \& Skibsted, L. H. (2006). ESR Spectroscopy for the Study of Oxidative Processes in Food and Beverages. In G. A. Webb (Ed.). Modern Magnetic Resonance (pp. 1861-1866). Dordrecht: Springer, Netherlands.

Barriuso, B., Astiasarán, I., \& Ansorena, D. (2013). A review of analytical methods measuring lipid oxidation status in foods: A challenging task. European Food Research and Technology, 236(1), 1-15. https://doi.org/10.1007/s00217-012-1866-9.

Berton, C., Ropers, M.-H., Viau, M., \& Genot, C. (2011). Contribution of the Interfacial Layer to the Protection of Emulsified Lipids against Oxidation. Journal of Agricultural and Food Chemistry, 59(9), 5052-5061. https://doi.org/10.1021/jf200086n.

Berton-Carabin, C. C., Ropers, M.-H., \& Genot, C. (2014). Lipid Oxidation in Oil-in-Water Emulsions: Involvement of the Interfacial Layer. Comprehensive Reviews in Food Science and Food Safety, 13(5), 945-977. https://doi.org/10.1111/1541-4337.12097.

Boerekamp, D. M. W., Andersen, M. L., Jacobsen, C., Chronakis, I. S., \& García-Moreno, P. J. (2019). Oxygen permeability and oxidative stability of fish oil-loaded electrosprayed capsules measured by Electron Spin Resonance: Effect of dextran and glucose syrup as main encapsulating materials. Food Chemistry, 287, 287-294. https://doi.org/10.1016/j.foodchem.2019.02.096.

Cui, L., Lahti, P. M., \& Decker, E. A. (2016). Evaluating Electron Paramagnetic Resonance (EPR) to Measure Lipid Oxidation Lag Phase for Shelf-Life Determination of Oils. Journal of the American Oil Chemists' Society, 94(1), 89-97. https://doi.org/10.1007/ s11746-016-2927-1.

Frankel, E. N. (2005). Free radical oxidation. Lipid Oxidation (pp. 15-24). (2nd ed.). Dundee, Scotland: Oily Press.

Janzen, E. G., Krygsman, P. H., Lindsay, D. A., \& Haire, D. L. (1990). Detection of Alkyl, Alkoxyl, and Alkyperoxyl Radicals from the Thermolysis of Azobis(isobutyronitrile) by ESR/Spin Trapping. Evidence for Double Spin Adducts from Liquid-Phase Chromatography and Mass Spectroscopy. Journal of American Chemical Society, 112, 8278-8284.

Laguerre, M., Tenon, M., Bily, A., \& Birtić, S. (2020). Toward a Spatiotemporal Model of Oxidation in Lipid Dispersions: A Hypothesis-Driven Review. European Journal of Lipid Science and Technology, n/a(n/a), 1900209. https://doi.org/10.1002/ejlt. 201900209.

Merkx, D. W. H., Delić, F., Wierenga, P. A., Hennebelle, M., \& van Duynhoven, J. P. M. (2019). 31P NMR assessment of the phosvitin-iron complex in mayonnaise. Magnetic Resonance in Chemistry, 57(9), 540-547. https://doi.org/10.1002/mrc.4808.

Merkx, D. W. H., Hong, S., Ermacora, A., \& van Duynhoven, J. (2018). Rapid Quantitative Profiling of Lipid Oxidation Products in a Food Emulsion by 1H NMR. Analytical Chemistry, 90(7), 4863-4870. https://doi.org/10.1021/acs.analchem.8b00380.

Mozuraityte, R., Rustad, T., \& Storro, I. (2008). The Role of Iron in Peroxidation of Polyunsaturated Fatty Acids in Liposomes. Journal of Agricultural and Food Chemistry, 56, 537-543.

Schaich, K. M. (2012). Thinking outside the classical chain reaction box of lipid oxidation. Lipid Technology, 24(3), 55-58. https://doi.org/10.1002/lite.201200170.

Schaich, K. M., Xie, J., \& Bogusz, B. A. (2017). Thinking outside the classical chain reaction box of lipid oxidation: Evidence for alternate pathways and the importance of epoxides. Lipid Technology, 29(9-10), 91-96. https://doi.org/10.1002/lite. 201700025.

Silvagni, A., Franco, L., Bagno, A., \& Rastrelli, F. (2010). Thermoinduced Lipid Oxidation of a Culinary Oil: A Kinetic Study of the Oxidation Products by Magnetic Resonance Spectroscopies. Journal of Physical Chemistry A, 114, 10059-10065.

Skiera, C., Steliopoulos, P., Kuballa, T., Holzgrabe, U., \& Diehl, B. (2012). 1H NMR approach as an alternative to the classical p-anisidine value method. European Food 
Research and Technology, 235, 1101-1105.

Steenhorst-Slikkerveer, L., Louter, A., Janssen, H.-G., \& Bauer-Plank, C. (2000). Analysis of nonvolatile lipid oxidation products in vegetable oils by normal-phase high-performance liquid chromatography with mass spectrometric detection. Journal of the American Oil Chemists' Society, 77(8), 837. https://doi.org/10.1007/s11746-0000134-1.

Stoll, S., \& Schweiger, A. (2006). EasySpin, a comprehensive software package for spectral simulation and analysis in EPR. Journal of Magnetic Resonance, 178(1), 42-55. https://doi.org/10.1016/j.jmr.2005.08.013.

Thomsen, M. K., Jacobsen, C., \& Skibsted, L. H. (2000). Mechanism of initiation of oxidation in mayonnaise enriched with fish oil as studied by electron spin resonance spectroscopy. European Food Research and Technology, 211, 381-386.

Thomsen, M. K., Kristensen, D., \& Skibsted, L. H. (2000). Electron spin resonance spectroscopy for determination of the oxidative stability of food lipids. Journal of American Oil Chemical Society, 77(7), 725-730.

Thomsen, M. K., Vedstesen, H., \& Skibsted, L. H. (1999). Quantification of Radical
Formation in Oil-in-Water Food Emulsions by Electron Spin Resonance Spectroscopy. Journal of Food Lipids, 6, 149-158.

Velasco, J. N., Andersen, M. L., \& Skibsted, L. H. (2004). Evaluation of oxidative stability of vegetable oils by monitoring the tendency to radical formation. A comparison of electron spin resonance spectroscopy with the Rancimat method and differential scanning calorimetry. Food Chemistry, 85(4), 623-632. https://doi.org/10.1016/j. foodchem.2003.07.020.

Velasco, J., Andersen, M. L., \& Skibsted, L. H. (2005). Electron Spin Resonance Spin Trapping for Analysis of Lipid Oxidation in Oils: Inhibiting Effect of the Spin Trap alpha-Phenyl-N-tert-butylnitrone on Lipid Oxidation. Journal of Agricultural and Food Chemistry, 53, 1328-1336.

Vicente, M. L., Empis, J. A., Deighton, N., Glidewell, S. M., Goodman, B. A., \& Rowlands, C. C. (1998). Use of EPR and ENDOR spectroscopy in conjunction with the spin trapping technique to study the high-temperature oxidative degradation of fatty acid methyl esters Journal of the Chemical Society. Perkin Transactions, 2, 449-454. 